\title{
Numerical techniques for the analysis of polygenes sampled from natural populations
}

\author{
J.N. THOMPSON, jr. *, Jenna J. HELLACK *, **, G.D. SCHNELL * \\ * Department of Zoology, University of Oklahoma, Norman, Oklahoma \\ 73019, U.S.A. \\ ** Department of Biology, Central State University, Edmond, Oklahoma \\ 73034, U.S.A.
}

\begin{abstract}
Summary
While polygenic factors contribute to almost every aspect of development, the small quantitative contributions of individual polygenic loci are typically difficult to analyze. A number of studies under controlled laboratory environments have shown that a large proportion of the variation in a quantitative trait can often be traced to a relatively small number of segregating loci. In natural populations, the establishment of a series of isofemale strains provides a sample of the segregating genetic variation. Furthermore, in each strain, the segregating genetic component is dramatically simplified. In this paper we describe numerical techniques than can be used to summarize interstrain differences based upon detected patterns of genetic segregation in isofemale lines. These techniques include UPGMA cluster analysis, K-group cluster analysis, and principal coordinates analysis. Distances between phenotypic distributions of isofemale line progeny are provided by the Kolmogorov-Smirnov (K-S) two-sample test. Overall, the use of K-S distances in conjunction with clustering and ordination techniques shows great promise in assisting population geneticists in the identification of strains with similar genetic characteristics.
\end{abstract}

Key words : Quantitative variation, simulation, cluster analysis, Drosophila melanogaster.

\section{Résumé}

Méthodes numériques pour l'analyse de polygènes échantillonnés dans des populations naturelles

Alors que les facteurs polygéniques contribuent à presque tous les aspects du développement, les faibles contributions individuelles des locus polygéniques sont difficiles à analyser.

Plusieurs études, conduites dans des environnements contrôlés en laboratoire, ont montré qu'une proportion importante de la variabilité d'un caractère quantitatif pouvait souvent être rapportée à un nombre relativement faible de locus en ségrégation. Dans les populations naturelles, l'établissement de séries de lignées isofemelles constitue un échantillonnage de la variabilité génétique. De plus, dans chaque lignée, la ségrégation des composantes génétiques est considérablement simplifiée. Dans cet article, on décrit des techniques numériques qui peuvent être utilisées pour décrire simplement des différences 
entre souches, en se fondant sur les profils de ségrégation génétique dans les lignées isofemelles. Ces méthodes sont fondées sur un indice de distance entre les distributions phénotypiques des descendances des lignées isofemelles, calculé d'après le test (K-S) de KOLMOGOROV-SMIRNOV.

Deux techniques de classification hiérarchique et une analyse en composantes principales sont mises en œuvre. D'une façon générale, l'utilisation conjointe des distances K-S et des techniques d'analyse de données semble très prometteuse pour aider les généticiens à identifier des souches possédant des caractéristiques génétiques semblables.

Mots clés : Variation quantitative, simulation, classification automatique, Drosophila melanogaster.

\section{Introduction}

The genetic makeup of a natural population can be characterized by the allele frequencies in its gene pool. This has been done most thoroughly for genes whose protein products are known or whose DNA has been cloned (LEWONTIN, 1974 ; HARTL, 1980). But such obvious genetic variants often play a smaller role in the adaptability of a population than do the much more numerous polygenic factors that contribute to essentially every aspect of development (Hosgood \& PARsons, 1967 ; THOMPSON, 1975 ; Spiess, 1977 ; Parsons, 1980 ; HoffmanN et al., 1985). Unfortunately, the small quantitative contributions of polygenic loci are often hard to analyze individually. With this limitation in mind, however, it is important to look for ways to characterize the polygenic component of the gene pool with a degree of precision similar to that available for loci having larger phenotypic effects (THOMPson \& THODAY, 1979 ; Parsons, 1980).

Studies under controlled laboratory environments have repeatedly shown that a large proportion of the variation in a quantitative trait can often be traced to a small number of segregating loci. Indeed, under appropriately controlled genetic and environmental conditions, individual polygenic alleles can be identified and mapped (Thompson \& ThodAY, 1979 ; Schnee \& Thompson, 1984). This encourages us to be optimistic about similar studies in less controlled conditions. While polygenic loci are readily masked by environmental factors and other gene effects, a few contribute significantly to the developmental expression of a trait and, therefore, should be recognizable even in natural populations.

Here we describe a new approach to the analysis of natural polygenic variation, and we evaluate its sensitivity under simulated and experimental conditions. Our approach involves statistical techniques originally developed by numerical taxonomists interested in evaluating numerical differences among geographical or temporal population samples. But within populations, there is analogous variation among the genomes of individuals. This individual variation can be categorized by comparing the segregational patterns shown in the progeny of standardized crosses. Whereas the numerical taxonomist typically evaluates differences among species or among populations, we are interested in assessing differences across families within the same population. Our primary objective is to categorize family samples into genetically similar groups. From these groups, it is then possible to deduce important information about the polygenic makeup of the sampled population. 


\section{Materials and methods}

Isofemale strains are established from single inseminated females sampled from a natural population (PARsons, 1980). Each set of offspring therefore carries a limited sample of the genetic variation segregating in the original population. If mating is at random with respect to the polygenic loci of interest, the genetic makeup of isofemale strains will differ as a function of the gene frequencies in the population and the probabilities of each type of mating.

In this paper we describe methods that categorize isofemale strains into appropriate segregational classes. Then, from the proportion of strains in each class, we can estimate the polygenic allele frequencies in the sampled natural population. In practice, segregation in a tested strain is detected by crossing individual males of the strain to females from an inbred standard strain. In such a cross, the phenotypic differences among their progeny are due to genetic variation among male gametes. We assume that minor environmental influences act at random on the offspring. The breeding programs involved in such an analysis are discussed in later sections (see also Thompson \& Mascie-Taylor, 1985).

In the statistical analysis of differences among strains, the first step is to calculate a measure of "distance" between each pair of strains, which yields a matrix of all interstrain distances. Trends and groupings represented in such a matrix can be complex, particularly when many strains are involved. It is therefore useful to employ additional techniques that summarize the interstrain associations. We selected the following 3 techniques for this purpose : (1) UPGMA cluster analysis ; (2) K-group cluster analysis; and (3) principal coordinates analysis.

\section{A. Distance measure}

We employed a $Z$-value resulting from the Kolmogorov-Smirnov two-sample test (SIEGEL, 1956 ; SOKAL \& ROHLF, 1981) as a measure of the dissimilarity of any pair of isofemale lines. The Kolmogorov-Smirnov two-sample test (hereafter referred to as the K-S test) is used to evaluate whether 2 independent samples have been drawn from the same population or from populations with the same distribution. It is sensitive to differences in the original distributions from which the samples are drawn, such as differences in location (central tendency), dispersion, or skewness (SIEGEL, 1956). The test is based on the unsigned differences between the relative cumulative frequency distributions of the two samples, which is a measure of the agreement of the 2 cumulative distributions. If 2 samples have been drawn from the same population, then the cumulative distributions of the 2 samples should show only random deviations from the distribution of the population.

First, the maximum difference $(D)$ is calculated between the 2 cumulative frequency distributions. The $Z$-value is then obtained from the following formula to adjust for samples sizes :

$$
Z=D^{*} \operatorname{SQRT}\left\{\left(X_{\mathrm{n}}{ }^{*} X_{\mathrm{m}}\right) /\left(X_{\mathrm{n}}+X_{\mathrm{m}}\right)\right\},
$$

where $X_{\mathrm{n}}$ and $X_{\mathrm{m}}$ are the numbers of observations in the 2 distributions being compared. The Statistical Package for the Social Sciences (SPSS, INC., 1983) 
calculates the $Z$-values and the given probability levels. In our case, the $Z$-value was derived as a distance (i.e., dissimilarity) measure between 2 strains. We thus calculated it for all strain pairs to produce a matrix of pair-wise distance values.

\section{B. UPGMA cluster analysis}

As one way of summarizing differences between all pairs of isofemale lines, hierarchical cluster analyses were performed on a matrix of K-S $Z$-values for all pairs. Specifically, we employed the unweighted pair-group method using arithmetic averages (UPGMA) as the clustering technique (SNEATH \& SOKAL, 1973 ; ROHLF et al., 1982). Cophenetic correlation coefficients were computed to indicate the degree to which $Z$-values in the resulting dendrogram were concordant with the original $Z$-values.

The use of this analysis assumes the presence of clusters. The acknowledgment of this assumption is important because this, like all such analyses, will show clusters of data sets even if there is no biological significance. One must therefore be careful to keep the biological context and limitation clearly in mind throughout any analysis.

\section{K-group cluster analysis}

We also obtained clustering results using a $K$-group method called functionpoint cluster analysis (KATZ \& ROHLF, 1973). Isofemale lines are assigned to a series of subgroups or clusters at a specific level. The computer program we used was described by RoHLF et al. (1982). The value for the $w$-parameter used in the functionpoint clustering method was varied, with each showing the clusters at a particular level.

Results from a series of these levels can be viewed and interpreted as a hierarchical series of clusters, although the results at one level of similarity are computed without knowledge of those produced at a higher or lower level. Thus, it is possible to have a hierarchical classification that is not fully nested (i.e., one isofemale line might be a member of one cluster at one level of dissimilarity and of another cluster at a slightly different level).

The results from this type of clustering can be represented in a generalized skyline diagram (WIRTH et al., 1966). The isofemale lines are listed side-by-side along the $X$-axis, and $w$-values on the $Y$-axis, with values arranged low to high from top to bottom. On a line in the diagram for a particular $w$-value, isofemale lines in the same cluster can be assigned a cluster number. In this way it is easy to identify cluster members and to determine how many clusters are present at a particular level of dissimilarity.

\section{Principal coordinates analysis}

Ordination techniques can also be used to summarize information about relationships within a series of organisms (in this case, isofemale lines). Often it is desirable to summarize such associations in two- or three-dimensional representations, 
even though the relationships are multivariate in nature. Such summaries can aid workers in the inspection and interpretation of their data. One advantage of ordination techniques over clustering techniques is that they make no assumption about the presence of clusters in the data. Clusters, if present, will be depicted. On the other hand, if a more or less continuous distribution of points is the case, then the resulting diagram will reflect such a pattern.

The techniques described earlier produce a matrix of dissimilarities for all pairs of isofemale lines. Principal coordinates analysis, developed by Gower (1966), can be used to summarize relationships among these lines. It transforms a matrix of distances between objects (e.g., isofemale line genotypes) into scalar product form so that the objects can be represented in two- or three-dimensional scatter plots. The Numerical Taxonomy System of Multivariate Statistical Programs (NT-SYS; RoHLF et al., 1982) has a program that carries out the appropriate calculations.

\section{E. Comparison of dissimilarity matrices}

Environmental factors can affect our ability to identify genetically similar strains. To test the importance of such factors, one can analyze pairs of distance matrices in which one matrix (for simulated data) incorporates no environmental influences while the other has a specified level of random phenotypic variation. The Mantel procedure (MANTEL, 1967) is used to determine whether interstrain differences, with and without environmental variance added, were statistically associated in a linear manner. The observed association between sets of interstrain differences is tested relative to their permutational variance, and the resulting statistic is compared against a standard normal distribution. Examples of the test have been provided by Douglas \& ENDler (1982) and Schnell et al. (1985). Calculations were performed using GEOVAR, a set of computer programs written by David M. Mallis and provided by Robert R. Sokal.

The matrix correlation (SNEATH \& SOKAL, 1973) was also computed between pairs of matrices. Unfortunately, the statistical significance of these coefficients cannot be determined with conventional tests. The correlation is based upon associations between all pairs of strains, and these are not statistically independent. In spite of this, these correlations are useful descriptive statistics that indicate the degree to which corresponding interstrain distance values are associated. In later sections, we have plotted correlations values, but we have used Mantel tests to evaluate statistical significance.

\section{Structure and assumptions of the model}

The polygenic loci that contribute most significantly to the genetic diversity in a population are likely to be highly polymorphic. Furthermore, individual polygenic loci can have quantitatively different effects and their expression depends upon the relative importance of environmental factors acting during development. These charasteristics are built into the assumptions of our gene pool sampling procedure using isofemale strains. Sampling of hypothetical isofemale strains was simulated according to the steps outlined in figure 1. 


\section{NATURAL GENE POOL}
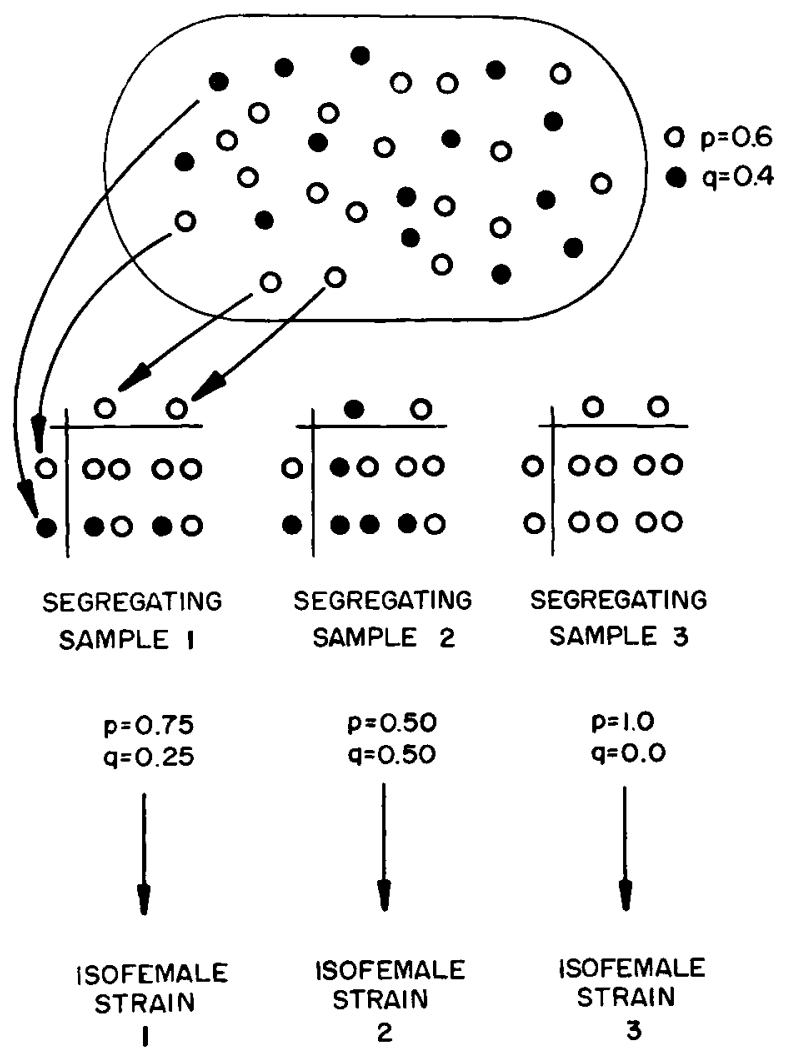

$p=1.0$

$q=0.0$

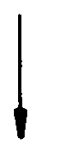
ISOFEMALE STRAIN
3

FIG. 1

Steps followed to produce isofemale lines in simulations.

For this simulation, we assume that there are 2 major polygenic alleles or linked complexes segregating in the gene pool. Each isofemale line derived from this pool carries a sample of alleles, ranging from one extreme to the other (from $p=1.0$ to $q=1.0$ ). The relative frequency of each type of isofemale line, however, will be a function of the relative frequency of each allele. In the gene pool in figure 1, for example, the number of isofemale strains segregating high frequencies of the "white " allele would be greater than the number with high frequencies of the "dark" allele. Furthermore, the proportion of "white" homozygotes among the progeny in sample 1 would be greater than in sample 2 . This theoretically allows one to distinguish genotypic differences, even among phenotypically similar strains. Consequently, by evaluating the patterns of segregation within a sample of isofemale strains, one can attempt to reconstruct the allelic composition of the original gene pool. 
This approach to dissecting the polygenic makeup of a natural population is dependent upon the following assumptions. First, the quantitative trait is influenced by a relatively small number of contributing processes (cf. THомpson, 1975). The phenotypic variation in sternopleural bristle number, for example, can typically be traced to a relatively small number of segregating alleles (THOMPSON \& THODAY, 1974), while a more complex trait, such as body weight or size (FALCONER, 1981), cannot. Yet, the composite quantitative trait «body weight " can be refined to focus upon one or a small number of contributing processes, such as muscle mass (cf. SPICKETT, 1963 ; SPICKETT et al., 1967). In this way polygenic segregation, even in a superficially complex quantitative trait, is potentially open to detailed analysis.

Phenotypic expression is also influenced by uncontrolled environmental factors that can enhance or suppress the action of genetic factors during development. Environmental factors do not always mask polygenic effects (THODAY \& THOMPson, 1976 ; ThOMPSON \& Hellack, 1982).

A second key assumption is that polygenic loci behave in a normal Mendelian fashion. They are not mobile genetic elements, unique components of heterochromatin, or some other novel genetic factor. Polygenes are simply assumed to be minor alleles, or isoalleles, of otherwise familiar genetic loci (THOMPsoN, 1975, 1977).

Third, matings are assumed to be at random with respect to the polygenic loci of interest and, in the present simulation, each individual mates only once. The assumption of single mating is clearly a simplifying assumption that will not necessarily hold in all populations (Mrlkman \& Zeitler, 1974 ; Gromko \& Pyle, 1978). In addition, mutation and selection are considered to be negligible. We shall discuss the consequences of relaxing these assumptions elsewhere.

Finally, we assume that a genetically homogeneous strain is available to serve as a standard in the analysis of segregational patterns. Such standard strains are common in genetically well-known organisms, and strains of satisfactory homogeneity can be produced by artificial selection in many species. The use of this standard is explained below.

\section{Analysis of polygenic segregational patterns}

We will first outline the sequence of analysis using a hypothetical example. The hypothetical standard for this example is homozygous for « - " alleles (MATHER \& JINKS, 1982) and has low expression of the character (e.g. low sternopleural bristle number in Drosophila). In our model, the «- - alleles add nothing to the baseline phenotype, while each " + » allele adds an increment of 2 units. The baseline value was set at 10 phenotypic units to allow random environmental factors to reduce phenotypic expression below that produced by a homozygous " - " genotype. This is analogous to studying the polygenic influences of enhancer and suppressor alleles acting upon a selected line of $D$. melanogaster having an average of 10 bristles. Scaled stochastic environmental effects produced additional variation in all phenotypes. Finally, in order to simplify graphical presentations, we arranged individual phenotypes into 25 classes (class $1=9.01-9.25$ units, class $2=9.26-9.50$, and so forth). 
In order to test the degree of segregation in a single isofemale line, several single-pair matings are made between a standard genetic strain and the isofemale strain. For example, 25 single-pair crosses of standard females to males from the tested line yield 25 sets of progeny that differ from one another only when they inherit different segregating alleles from the tested males. Phenotypic distributions from 7 representative isofemale strains are shown in figure 2 .

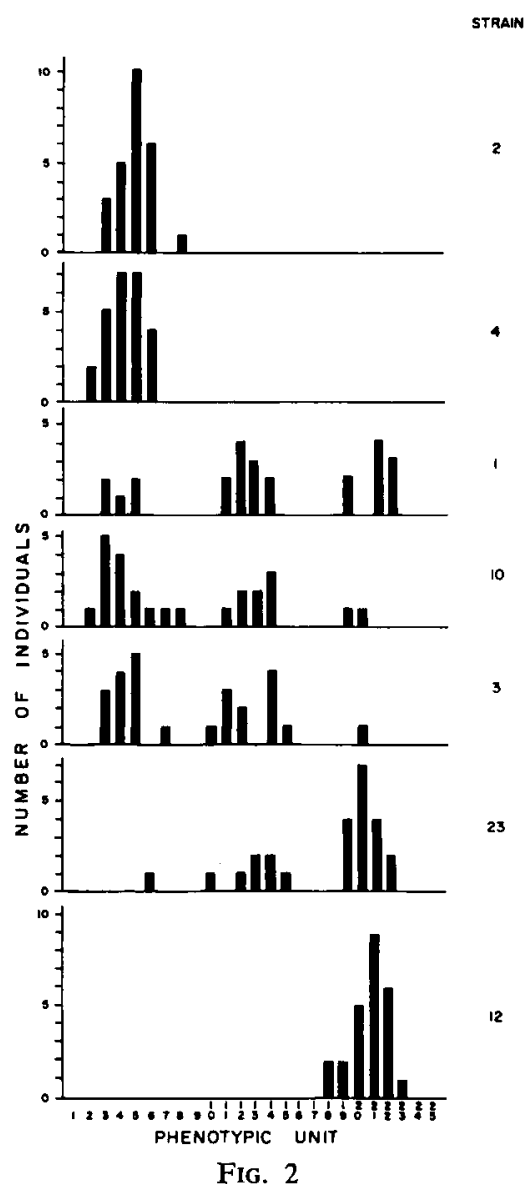

Phenotypic distributions for 7 hypothetical segregating isofemale strains.

Strains 2 and 4 are homozygous for the "low " allele $\left(\mathrm{A}^{2}\right)$. The 25 sets of progeny produced by crossing males from these strains to the \& low » standard are all phenotypically "low ». Strain 12, on the other hand, is homozygous for the " high " allele $\left(A^{1}\right)$. All of the progeny from the standard cross have inherited the $A^{1}$ allele from the father and are, therefore, heterozygous $A^{1} A^{2}$. The remaining strains are segregating for both alleles (table 1). 
TABLE 1

Parental genotypes in a sample of seven hypothetical isofemale strains.

\begin{tabular}{|c|c|c|c|c|}
\hline \multirow{2}{*}{ Strain } & \multicolumn{2}{|c|}{ Genotypes } & \multicolumn{2}{|c|}{$\begin{array}{l}\text { Isofemale Allele } \\
\text { Frequencies }\end{array}$} \\
\hline & Paternal & Maternal & $A^{1}$ & $A^{2}$ \\
\hline 1 & $\mathbf{A}^{1} \mathbf{A}^{1}$ & $\mathrm{~A}^{2} \mathrm{~A}^{2}$ & 0.50 & 0.50 \\
\hline 2 & $A^{2} A^{2}$ & $\mathrm{~A}^{2} \mathrm{~A}^{2}$ & 0.00 & 1.00 \\
\hline 3 & $A^{1} A^{2}$ & $A^{2} A^{2}$ & 0.25 & 0.75 \\
\hline 4 & $\mathrm{~A}^{2} \mathrm{~A}^{2}$ & $A^{2} A^{2}$ & 0.00 & 1.00 \\
\hline 10 & $A^{1} A^{2}$ & $A^{2} A^{2}$ & 0.25 & 0.75 \\
\hline 12 & $A^{1} A^{1}$ & $A^{1} A^{x}$ & 1.00 & 0.00 \\
\hline 23 & $A^{1} A^{1}$ & $\mathrm{~A}^{1} \mathrm{~A}^{2}$ & 0.75 & 0.25 \\
\hline
\end{tabular}

As outlined in the methods section, the degree of similarity between pairs of strains was quantified by the K-S test. The resulting Z-values for all pairs of strains (table 2) provided the distances necessary to construct the UPGMA dendrogram shown in figure 3 . The cophenetic correlation coefficient of 0.76 indicates that the dendrogram is a reasonable summary of the relationships represented in the distance matrix, although there are some distortions of distances from the original matrix.

TABLE 2

Distance matrix produced by the Kolmogorov-Smirnoff two-sample test.

\begin{tabular}{r|c|c|c|c|c|c}
\hline \multirow{2}{*}{ Strain } & \multicolumn{5}{|c}{ Strain } \\
\cline { 2 - 7 } & 1 & 2 & 3 & 4 & 10 & 12 \\
\cline { 2 - 7 } 2 & 2.828 & & & & & \\
3 & 1.556 & 1.697 & & & & \\
4 & 2.828 & 1.131 & 1.838 & & & \\
10 & 1.414 & 1.556 & 0.849 & 1.697 & & \\
12 & 2.263 & 3.536 & 3.394 & 3.536 & 3.253 & \\
23 & 1.414 & 3.394 & 2.404 & 3.394 & 2.263 & 1.697 \\
\hline
\end{tabular}

Strains 2 and 4 cluster together and are more similar to strains 3 and 10 than to the other 3 strains. Strains 3 and 10 share the fact that they are segregating one $\mathrm{A}^{1}$ allele and three $\mathrm{A}^{2}$ alleles. For the remaining three strains, 1 and 23 join and then are combined with strain 12. Each of these has a low frequency of the $\mathrm{A}^{2}$ allele. Thus, the UPGMA cluster analysis appears sensitive to the segregating genetic differences in these simulated strains, in spite of environmental effects. The role of environment is considered in greater detail below. 


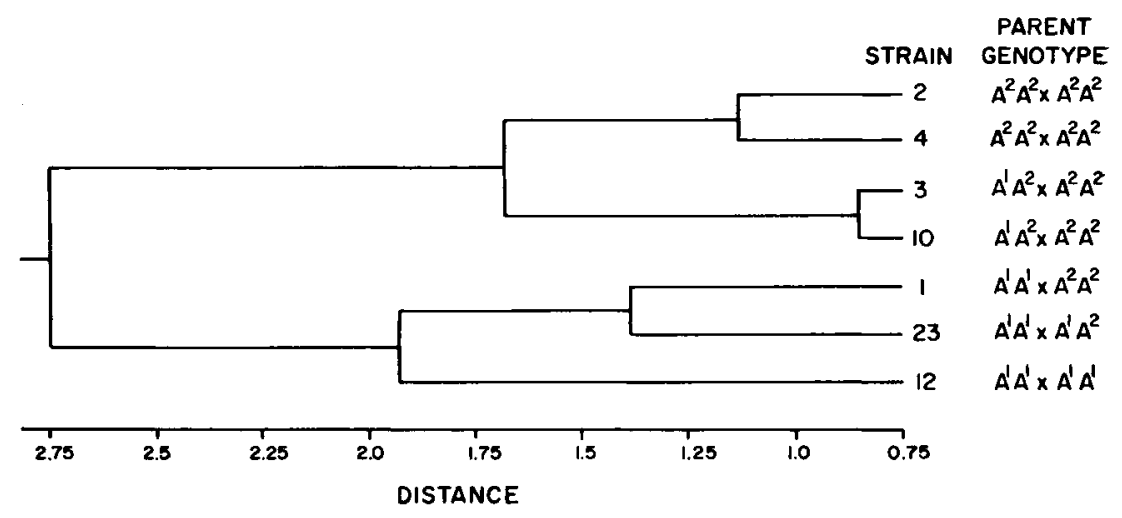

FIG. 3

UPGMA dendrogram summarizing associations among 7 strains based on $K-S$ distances.

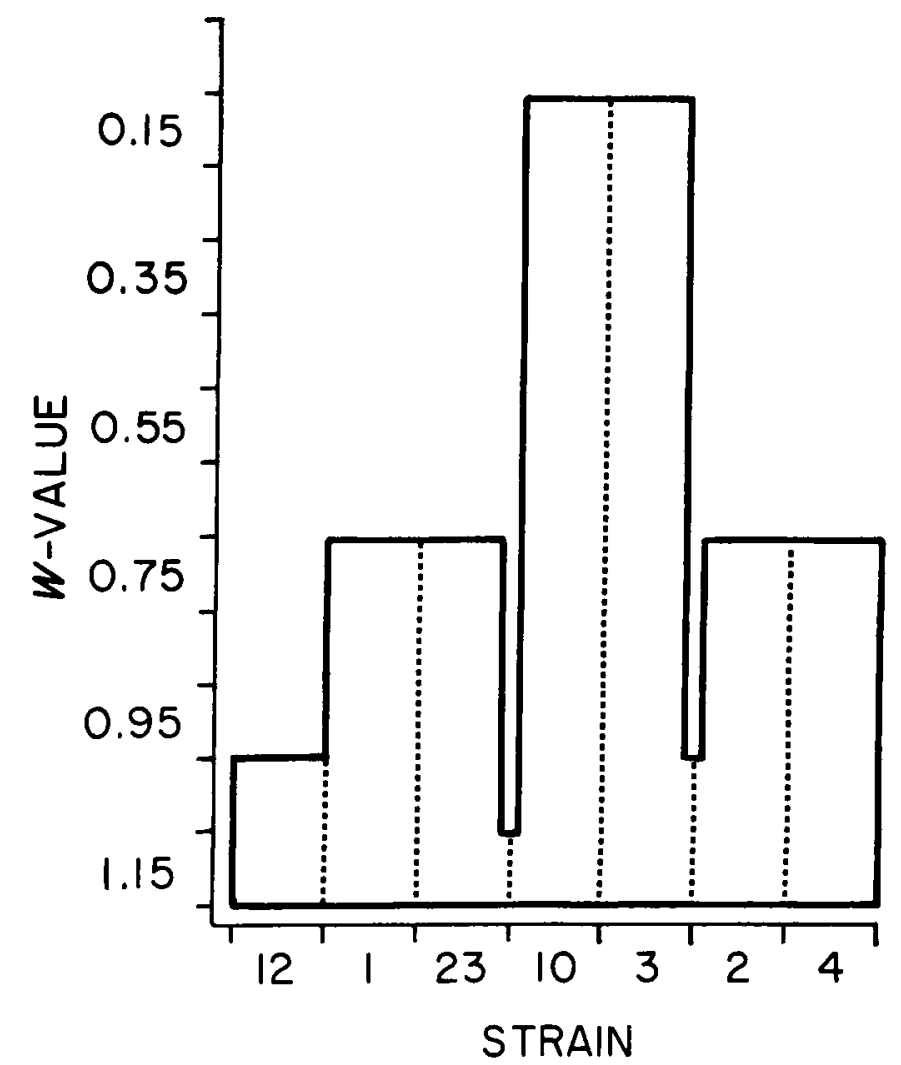

FIG. 4

Skyline diagram depicting associations among 7 strains

as determined using $\mathrm{K}$-group cluster analysis

to summarize associations indicated by $\mathrm{K}-\mathrm{S}$ distances. 
Similar groups were obtained using $K$-group cluster analysis (figure 4 ). Strains 3 and 10 are again the most closely associated, as indicated by the fact that they are still joined at the $0.15 w$-value. At a somewhat higher $w$-value of 0.75 , strains 1 and 23 group together, as do strains 2 and 4 . As reflected in the UPGMA dendrogram, strain 12 joins the $1-23$ group when the $w$-value is 1.05 , while the $3-10$ and 2-4 clusters are merged. All strains are combined into a single cluster when the $w$-value reaches 1.15 .

Principal coordinates analysis in another way of looking at the relationship among strains. The results are summarized in the plot in figure 5. Two axes account for much of the variation among strains. Axis I seems to separate strains on the basis of average strain phenotypes. Axis II separates strains 2, 4, and 12 (the non-segregating strains) from the others. The third axis (i.e., the heights of the spheres) may be responding to more subtle characteristics of the distributions, such as kurtosis, though it accounts for little of the variation among strains.

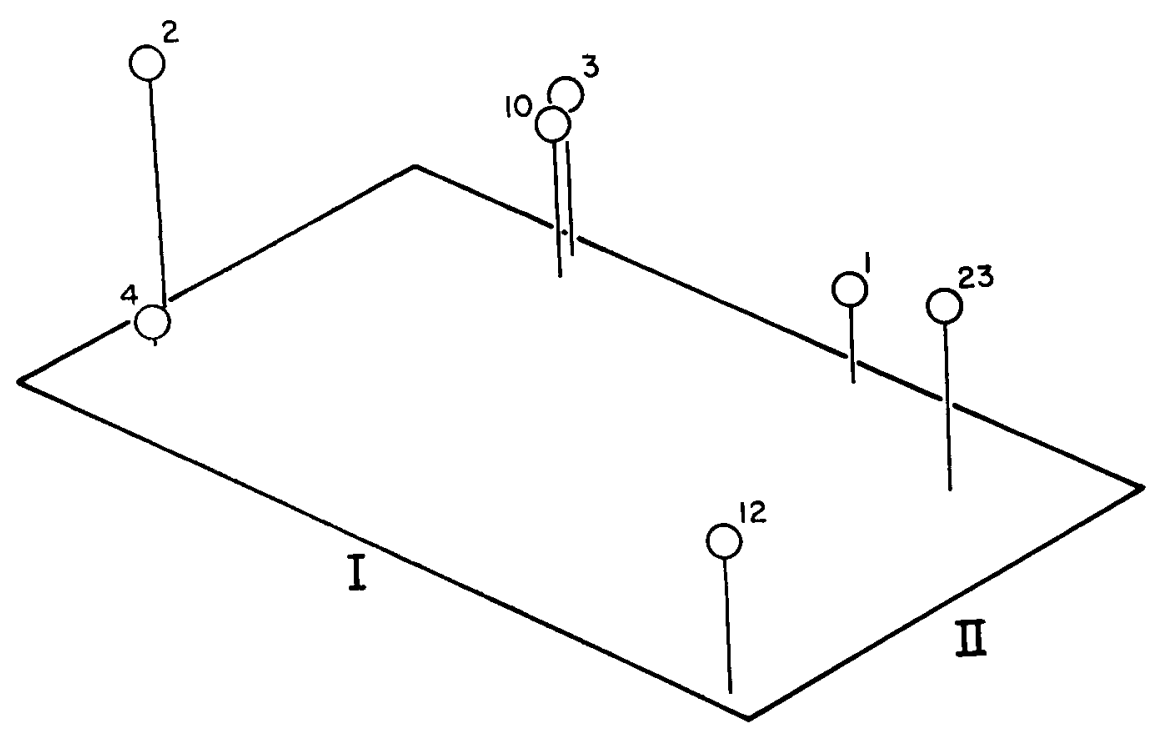

FIG. 5

Three-dimensional model showing similarities among 7 strains as determined by principal coordinates analysis of the $K-S$ distance matrix.

\section{Assessment of sensitivity}

\section{A. Interstrain differences and environmental variance}

Since environmental factors also affect the expression of polygenic traits, it is important to understand the sensitivity of techniques designed to identify clusters of genetically similar strains. The techniques presented in this paper operate on a matrix in interstrain distances. Thus, we have evaluated the changes in interstrain distances 
that result from adding random environmental variance to the segregating genetic component. The environmental component $\left(V_{\mathbf{E}}\right)$ was derived from a scaled distribution of random normal deviates. These scalar values are plotted along the $X$-axis in figure 6. At a value of 2.0, for example, the standard deviation of environmental effects is as large as the phenotypic influence of a single « high » polygenic allele.

Figure 6 shows the matrix correlations of interstrain distances that result from increasing environmental effects. Each correlation is calculated by comparing 2 matrices of $\mathrm{K}-\mathrm{S}$ values. In the initial matrix there is no environmental variance. This is contrasted with a comparable matrix in which a given level of random environmental effects has been included.
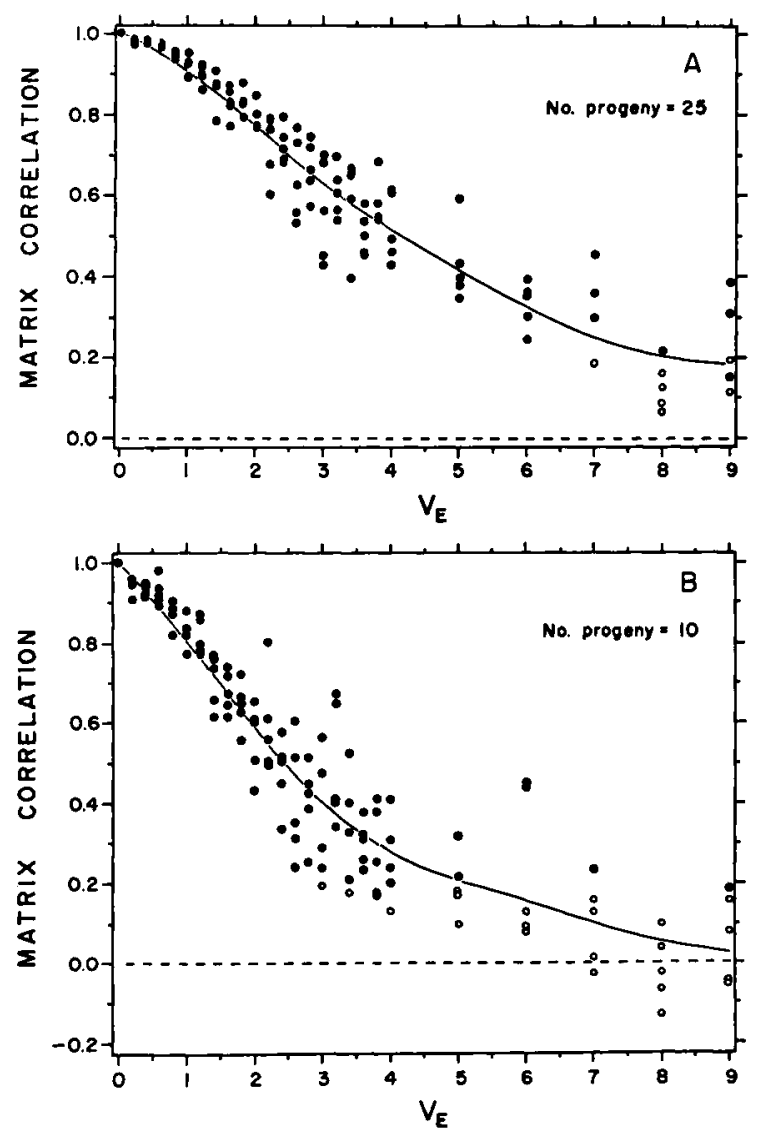

FIG. 6

Plots showing matrix correlations resulting from comparison of interstrain $K-S$ values.

Phenotypes with no added environmental variance component are compared with the interstrain values when increasing levels of environmental variance is included. In $(A)$ 25 progeny were scored in each of 25 strains per replicate; In (B), 10 progeny were scored. Each circle represents a single simulation run. A solid circle indicates a statistically significant $(\mathrm{P}<0.05)$ association as indicated by the Mantel test. An open circle indicates that there was no statistical association between the matrices with and without environmental variation. The plotted curve was produced by fitting a cubic spline that minimizes a linear combination of the sum of squares of the residuals of fit and the integral of the square of the second derivative (SAS Institute, 1981). 
When 25 progeny were used to provide an assessment of the phenotypic distribution within a strain (figure $6 \mathrm{~A}$ ), the matrix correlation dropped to about 0.80 when the environmental component of variance was 2.0. Not unexpectedly, the matrix correlation decreased as the environmental component increased. When the environmental component increased to 4 times the magnitude of a segregating polygenic allele (i.e., increased to 8.0 ), there was no longer a statistical concordance between the two K-S matrices, as measured by Mantel tests. Therefore, a conservative practical limit occurs when the magnitude of a segregating polygenic allele is about as large as the standard deviation of the random environmental effects (i.e., 2.0).

When intrastrain phenotypic distributions were estimated using only 10 progeny, matrix correlations dropped more rapidly as the environmental component increased (figue 6 B). Mantel tests assessing the differences between interstrain distance matrices indicated a lack of statistical concordance after the environmental component reached about 4.0. A comparison of figures $6 \mathrm{~A}$ and $6 \mathrm{~B}$ confirms that more reliable estimates of interstrain genetic differences are obtained when larger numbers of progeny are used to characterize intrastrain phenotypic distributions.

\section{B. UPGMA cluster analysis}

In order to see what effect the environmental factors have on our ability to distinguish the genotypes of our isofemale lines, we set up a population with 2 polygenic alleles. One (the $\mathrm{A}^{1}$ allele) was assigned a phenotypic effect of 2.0 , and the other allele $\left(A^{2}\right)$ had no effect on the base phenotype of 10 . The sampled population had allelic frequencies of $\mathrm{A}^{1}=\mathrm{A}^{2}=0.5$.

A total of 25 isofemales were randomly sampled from the population producing 25 strains. Figure 7 represents a UPGMA cluster analysis of these strains. Three levels of environmental variation were compared : (a) no environmental variation, (b) environmental variation equal to half the effect of allele $A^{1}$, and (c) environmental variation equal to the $A^{1}$ allele's effect.

In figure $7 \mathrm{~A}$, four clusters are evident. The first includes 4 isofemale strains $(1,12,17$, and 22). These represent strains produced from parents that are both homozygous for the "high » allele $\mathrm{A}^{1}$. The parents of strains in the second cluster, beginning with strain 2 and ending with strain 10 , have a total of two $A^{1}$ alleles, except strains 10 and 5 which have three $A^{1}$ alleles each. Cluster three (strains 4 through 11) has a single $A^{1}$ and three $A^{2}$ alleles. Cluster four contains only strain 13 , which is homozygous for the $\mathrm{A}^{2}$ allele. With the exception of strains 10 and 5 , the lines segregate into clusters according to their genetic makeup.

When the environmental component is 1.0 (figure $7 \mathrm{~B}$ ), similar groups of strains can still be identified using the UPGMA cluster analysis. The major difference was the placement of strain 10 . In the initial analysis (figure $7 \mathrm{~A}$ ), strain 10 was depicted as the most divergent strain in the second cluster and was one of the 2 strains in which the frequency of $\mathrm{A}^{1}$ was 0.75 . In figure $7 \mathrm{~B}$, strain 10 joins after the second and third cluster are combined. Other changes in strain associations occur within each of the clusters, although these reflect only minor modifications in the associations among genetically similar strains.

The main clusters are still present when the environmental component $(2.0)$ is equal to the effect of the "high" allele (figure $7 \mathrm{C}$ ). The main change involves 
strain 13, which was homozygous for allele $\mathrm{A}^{2}$. It now clusters with the strains which have only one $A^{1}$ allele, though it enters the cluster last. There are two strains (2 and 6 ) that change clusters. In spite of these modifications, we can still recognize the genetically different clusters of isofemale strains originally found in the absence of environmental effects.

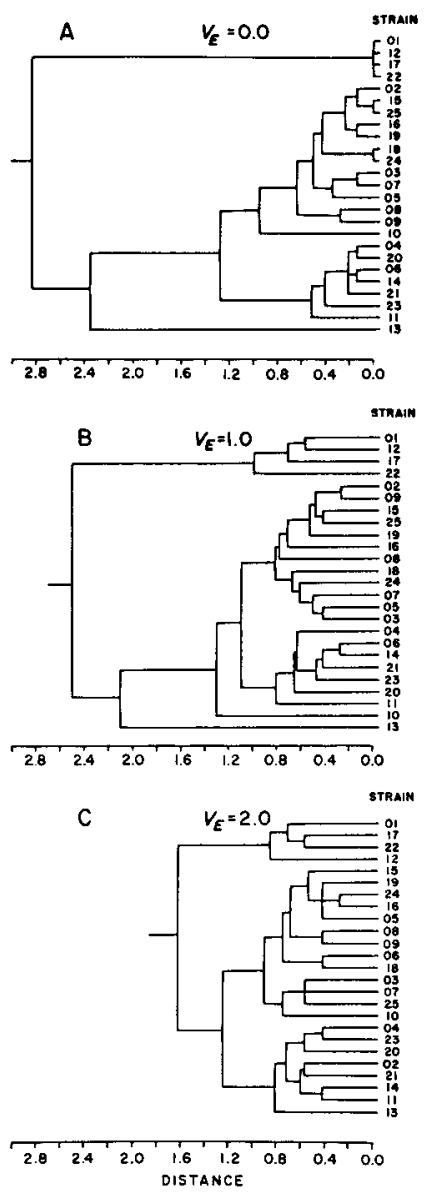

FIG. 7

UPGMA dendrograms summarizing associations among 25 strains based on $K-S$ distances as determined when the environmental variance component was $(A) 0.0,(B) 1.0$, and $(C) 2.0$.

The segregating polygene effect in each case was 2.0 .

Cophenetic correlations for the 3 dendrograms were $0.942,0.909$, and 0.760 , respectively.

C. K-group cluster analysis

A similar trend was found when groups were summarized using $K$-group cluster analysis (figure 8 ). With no environmental variance, the same group of 4 strains 
was included in cluster 1 . Strain 13 was separated into its own group when the $w$-value reached 0.75 . In addition, the second and third clusters found in the UPGMA cluster analysis were also identified when the $\boldsymbol{w}$-value was $\mathbf{0 . 4 5}$.
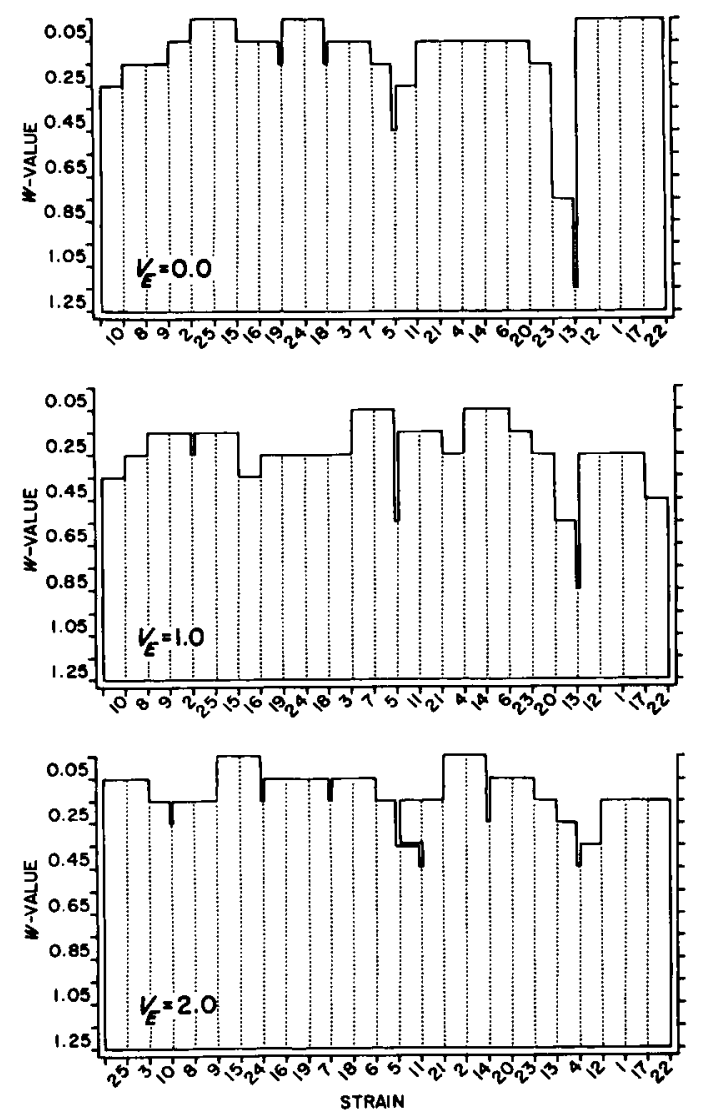

Fig. 8

Skyline diagrams indicating associations among 25 strains

as determined using K-group cluster analysis on K-S distances for the strains shown in fig. 7.

The same groups were found when the environmental component was set at 1.0 , although minor differences in interstrain associations are found within some of the 4 major groups. When environmental effects increase to 2.0 , much of the ability to resolve genetic differences was lost; groups were not found until the $w$-value was reduced to 0.45 . Note that strain 11 «switches " clusters from one level to another as the $w$-value is decreased from 0.45 to 0.35 , demonstrating that the clusters formed in this type of cluster analysis need not be nested. 


\section{Principal coordinates analysis}

Principal coordinates analyses are helpful in understanding the changes that occur among clusters due to increasing environmental variance. The same 4 clusters are clearly seen in the plot at the top of figure 9. In the absence of environmental influence, the differences among clusters can be totally summarized in 2 dimensions. The first component (I) separates strains on the basis of allelic frequency; the within-genotype variation results from stochastic sampling of parents in the simulation. From left to right in this figure, the frequency of the $A^{1}$ allele increases. Component $I$ therefore reflects the average strain phenotype.

Component II, on the other hand, separates strains on the basis of intrastrain variance in phenotype. The third component, represented by height of the spheres above the plane, is largely a function of stochastic environmental influences. Environmental variation also plays a role in the expression of components I and II, especially when $V_{E}$ becomes larger, as in the lower diagrams in figure 9.
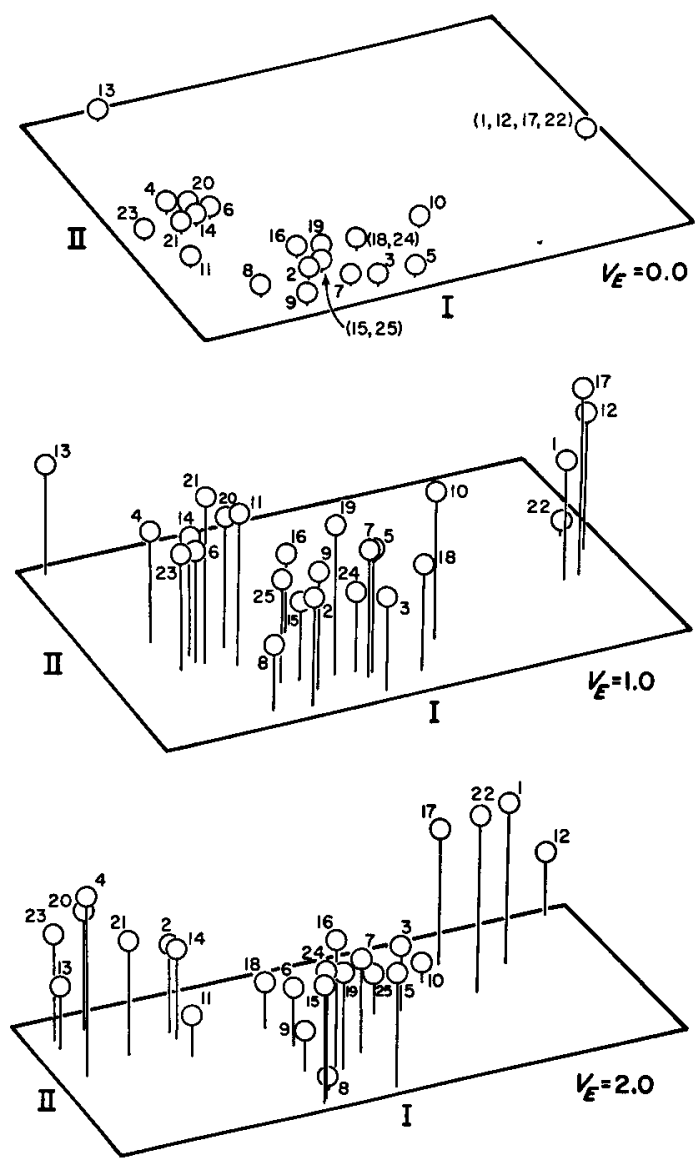

FIG. 9

Three-dimensional models indicating similarities among 25 strains as determined by conducting a principal coordinates analysis of $K-S$ distance as in fig. 7. 

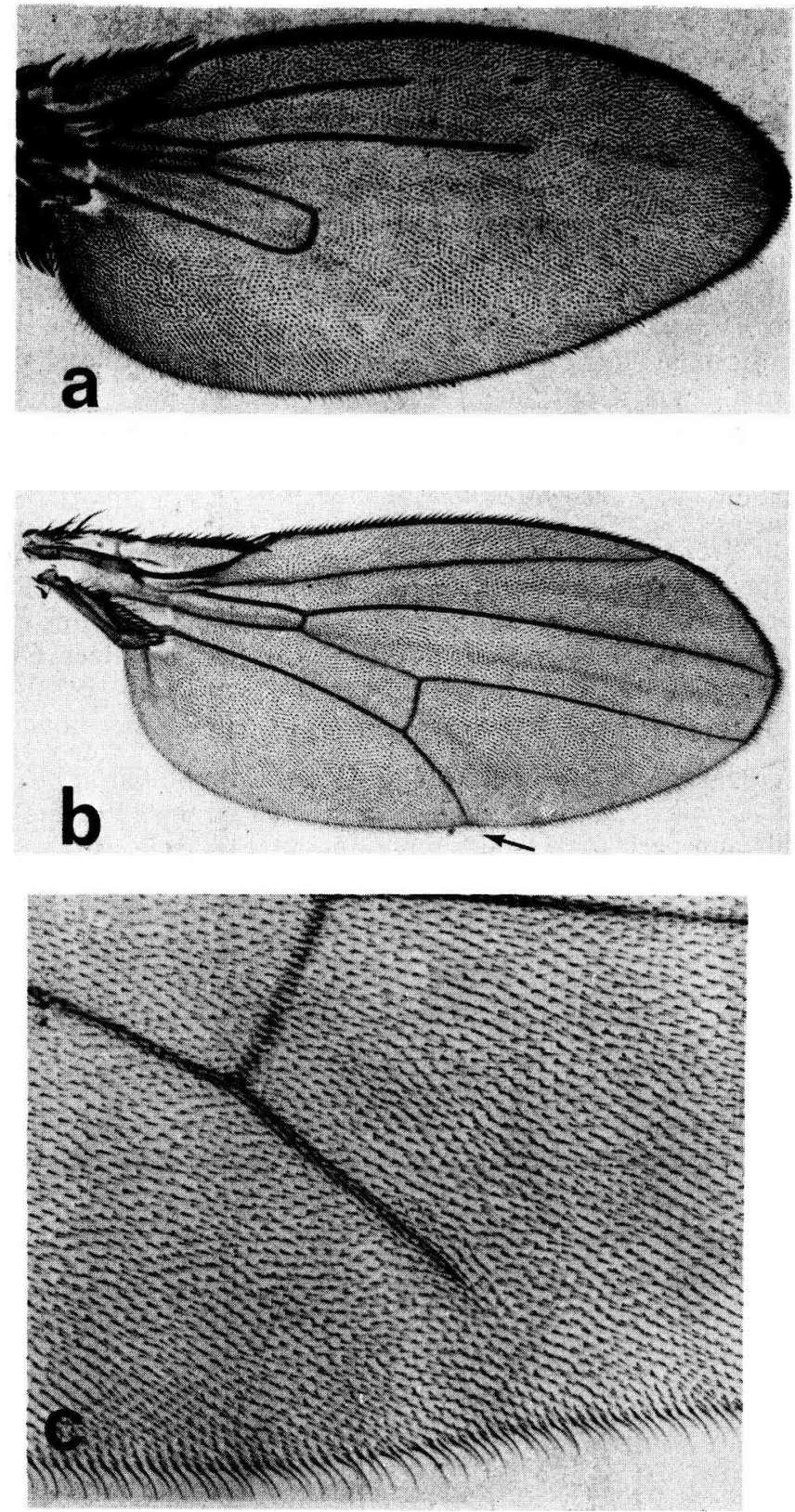

Fig. 10

Wings of Drosophila melanogaster.

(a) veinlet selected for shortened veins; (b) wild type with a complete L5 vein (indicated by arrow); (c) L5 gap in polygenic background allowing penetrance of heterozygous veinlet. 


\section{Experimental example and discussion}

The analyses we have described here can be used to characterize the major segregating components of a polygenic system under controlled environmental conditions. In contrast to the simplifying assumption of biometrical genetics (MATHER, 1943), the most critical assumption of our approach is that polygenic loci can differ significantly in the level of effect they have upon phenotypic expression. Many will have such small influences that they will be masked by random environmental factors. The effects of other polygenic loci will be comparatively large. Such loci will contribute significantly to selection responses favoring phenotypic change or stability. The experimental support for this view of polygene action is now quite extensive (see references in Thompson \& Thoday, 1979 ; Parsons, 1980 ; Mather \& Jinks, 1982). It is these major polygenic loci that we are most interested in identifying in a natural population.

The results from this simulated population demonstrate that major polygenic factors could theoretically be identified from a natural population. There have been other experimental attempts to detect polygenic factors in nature, such as that by Milkman (1970) and Boyer et al. (1973) using special selection lines. Other approaches call upon a variety of statistical (LANDE, 1981 ; ELSTON et al., 1978) and laboratory (Thompson \& Hellack, 1982 ; SchneE \& Thompson, 1985) techniques.

A major advantage of our approach using several multivariate techniques is that one can rapidly compare a large number of related strains. We are in the process of applying this approach to several simple quantitative traits in Drosophila melano-

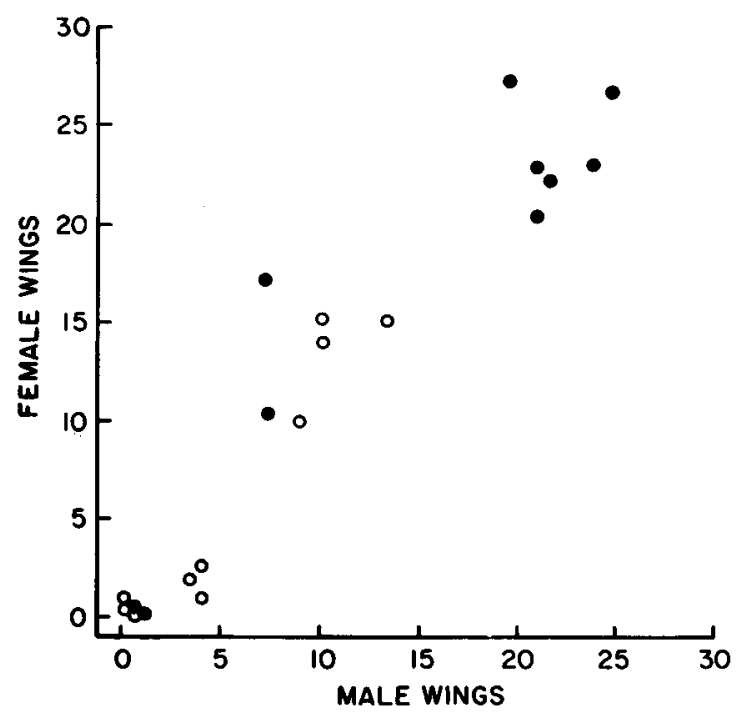

FIG. 11

L5 wing vein gap frequencies shown by the 10 replicates of isofemale line \# 1 (solid circles) and isofemale line \# 20 (open circles). 
gaster. One completed study (ThOMPSON \& MAscie-TAYLOR, 1985), however, confirms that these analyses work with real characters.

The polygenic system studied by THOMPSON \& MASCIE-TAYLOR (1985) was the set of modifiers of fifth longitudinal (L 5) vein development in $D$. melanogaster (figure 10). Males from each of 100 tested isofemale strains were mated to inbred selected females carrying the recessive mutant veinlet. $F_{1}$ progeny were scored for the frequency of L5 vein gaps in each cross. Segregation of high, intermediate, and low frequency gap lines was quite evident in many of the crosses (figure 11); six distinct clusters of strains were found. Tentative mapping of the vein modifiers was consistent with the interpretation that as few as 1 or 2 major polygenic L5 vein modifiers were segregating in the population.

Polygenic factors are an important, but poorly understood, component of a gene pool. Even limited success in determining allelic makeup of a natural population can add a valuable dimension to our understanding of population structure and adaptability. The use of multivariate techniques to analyze Kolmogorov-Smirnov Z-statistics shows promise as an aid in assessing allelic effects. The statistic simultaneously evaluates all types of differences (e.g., central tendency, dispersion, skewness, kurtosis) between distributions of phenotypes, rather than analyzing them separately. As an overall measure, the $Z$-statistic performed well. Its use could be combined with other techniques that decompose variation into separate components, particularly when we have a more complete understanding of which aspects of distributional differences are important for the effective identification of polygenic factors.

Received November 5, 1985.

Accepted February 24, 1986.

\section{Arknowledgements}

We thank F. James RoHLF for several useful suggestions on numerical techniques, Peter A. Parsons for his discussions of isofemale lines, and Daniel J. Hough for technical assistance. The illustrations were prepared by Laura KARCHER. This research was supported by the National Science Foundation under grant number BSR-8300025.

\section{References}

Boyer B.J., Parris D.L., Milkman R., 1973. The crossveinless polygenes in an Iowa population. Genetics, 75, 169-179.

Douglas M.E., ENDLER J.A., 1982. Quantitative matrix comparisons in ecological and evolutionary investigations. J. Theoret. Biol., 99, 777-795.

Elston R.C., Namboodiri K.K., Kaplan E.B., 1978. Resolution of major loci for quantitative traits. In : MoRTon N.E., Chung C.S. (ed.), Genetic epidemiology, 223-235, Academic Press, New York.

FALCONER D.S., 1981. Introduction to quantitative genetics, 2nd ed., 340 pp., Longman, London.

GOWER J.C., 1966. Some distance properties of latent root and vector methods used in multivariate analysis. Biometrika, 53, 325-338.

Gromko M.H., PYLE D.W., 1978. Sperm competition, male fitness, and repeated mating by female Drosophila melanogaster. Evolution, 32, 588-593. 
Hartl D.L., 1980. Principles of population genetics. 488 pp., Sinauer, Sunderland, Mass.

Hoffmann A.A., Nielsen K.M., Parsons P.A., 1985. Spatial variation of biochemical and ecological phenotypes in Drosophila : electrophoretic and quantitative variation. Heredity, 53 (in press).

HosGOOD S.M.W., Parsons P.A., 1967. The exploitation of genetic heterogeneity among the founders of laboratory populations of Drosophila prior to directional selection. Experientia, 23, 1066-1067.

KATZ J.O., RoHLF F.J., 1973. Function-point cluster analysis. Syst. Zool., 22, 295-301.

LANDE R., 1981. The minimum number of genes contributing to quantitative variation between and within populations. Genetics, 99, 541-553.

LEwonTIN R.C., 1974. The genetic basis of evolutionary change. 346 pp., Columbia Univ. Press, New York.

MANTEL N., 1967. The detection of disease clustering and a generalized regression approach. Cancer Res., 27, 209-220.

MATHER K., 1943. Polygenic inheritance and natural selection. Biol. Rev., 18, 32-64.

MATHER K., JinKS J.L., 1982. Biometrical genetics. 3rd ed., 396 pp., Chapman and Hall, London.

Milkman R., 1970. The genetic basis of natural variation in Drosophila melanogaster. Adv. Genet., 15, 55-114.

Milkman R., ZeITLER R.R., 1974. Concurrent multiple paternity in natural and laboratory populations of Drosophila melanogaster. Genetics, 78, 1191-1193.

PARSONS P.A., 1980. Isofemale strains and evolutionary strategies in natural populations. In : Hecht M.K., Steere W.C., Wallace B. (ed.), Evolutionary biology, vol. 13, 175-217, Plenum Press, New York.

RohlF F.J, KishPaUgh J., KIRK D., 1982. NT-SYS. Numerical taxonomy system of multivariate statistical programs. State Univ. New York, Stony Brook, New York.

SAS INSTITUTE, INC., 1981. SAS/Graph user's guide. 596 pp., SAS Institute, Inc., Cary, North Carolina.

SCHNEE F.B., THOMPSON J.N., Jr., 1984. Conditional neutrality of polygene effects. Evolution, 38, 42-46.

SCHNEE F.B., Thompson J.N., Jr., 1985. Conditional polygenic effects in the sternopleural bristle system of Drosophila melanogaster. Genetics, 108, 409-424.

SChNell G.D., WatT D.J., Douglas M.E., 1985. Statistical comparison of proximity matrices : applications in animal behaviour. Anim. Behav., 33, 239-253.

SIEGEL S., 1956. Nonparametric statistics for the behavioral sciences, $312 \mathrm{pp}$., McGraw-Hill Book Co., New York.

SNEATH P.H.A., SOKal R.R., 1973. Numerical taxonomy. 573 pp., W.H. Freeman and Co., San Francisco.

SOKAL R.R., RoHLF F.J., 1981. Biometry. 859 pp., W.H. Freeman and Co., San Francisco.

SPICKETT S.G., 1963. Genetic and developmental studies of a quantitative character. Nature, 199, 870-873.

SPICKeTt S.G., Shire J.G.M., Stewart J., 1967. Genetic variation in adrenal and renal structure and function. In : SPICKeTT S.G., SHIRE J.G.M. (ed.), Endocrine genetics. Mem. Soc. Endocrinology, vol. 15, 271-288 Cambridge Univ. Press, London.

SPIESS E.B., 1977. Genes in populations. $780 \mathrm{pp}$. John Wiley and Sons, New York.

SPSS INC., 1983. SPSS* user's guide. 806 pp., McGraw-Hill, New York.

THODAY J.M., THOMPSON J.N., Jr., 1976. The number of segregating genes implied by continuous variation. Genetica, 46, 335-344.

Thompson J.N., Jr., 1975. Quantitative variation and gene number. Nature, 258, 665-668.

THOMPSON J.N., Jr., 1977. Analysis of gene number and development in polygenic systems. Stadler Symposium, 9, 63-82.

Thompson J.N., Jr., HELlaCK J.J., 1982. Polygene segregation within an isofemale strain of Drosophila. Can. J. Genet. Cytol., 24, 235-241.

Thompson J.N., Jr., MASCIE-TAYLOR C.G.N., 1985. Detection of simple polygenic segregations in a natural population. Proc. Natl. Acad. Sci. U.S.A., 82, 8552-8556.

Thompson J.N., Jr., ThODAY J.M., 1974. A definition and standard nomenclature for \& polygenic loci ». Heredity, 33, 430-437.

Thompson J.N., Jr., ThodaY J.M., 1979. Quantitative genetic variation. 305 pp., Academic Press, New York.

Wirth M., Eastabrook G.F., Rogers D.F., 1966. A graph theory model for systematic biology with an example for the Oncidiinae (Orchidaceae). Syst. Zool., 15, 59-69. 